\title{
Flora of Danau Dusun Besar conservation forest in Bengkulu Province, Indonesia
}

\author{
WIRYONO $^{1, \bullet}$, ROHIDIN MERSYAH $^{2}$, MARISKA TARANTONA ${ }^{3}$ \\ ${ }^{1}$ Department of Forestry, Faculty of Agriculture, Universitas Bengkulu. Jl. WR Supratman, Bengkulu 38371A, Bengkulu, Indonesia. \\ Tel./fax.: +62-736-21170, Fax.: +62-736-21290, ^email: wiryonogood@yahoo.com; wiryonogood@unib.ac.id \\ ${ }^{2}$ Graduate Program in Natural Resource Management, Faculty of Agriculture, Universitas Bengkulu. J1. WR Supratman, Bengkulu 38371A, Bengkulu, \\ Indonesia \\ ${ }^{3}$ Natural Resource Conservation Agency of Bengkulu. Jl. Mahakam, Kota Bengkulu 38114, Bengkulu, Indonesia
}

Manuscript received: 17 September 2020. Revision accepted: 9 November 2020.

\begin{abstract}
Wiryono Mersyah R, Tarantona M. 2020. Flora of Danau Dusun Besar conservation forest in Bengkulu Province, Indonesia. Biodiversitas 21: 5640-5649. Few studies on flora of freshwater swamp forest in Sumatra have been published and accessible online. This study aimed to assess the composition and diversity of plant species and to describe the dominant species in conservation forest of Danau Dusun Besar Nature Reserve and Nature Tourism Park in Bengkulu which consists mostly of freshwater swamp forests and a lake. Data collection was done using a combination of quantitative methods with systematic plots and qualitative methods through observation. The quantitative data were analyzed to determine indexes of diversity, evenness and importance value. This study recorded 23 species of trees with diversity index $\left(\mathrm{H}^{\prime}\right)$ and evenness index $(\mathrm{E})$ of 0.29 and 0.09 , respectively, and recorded 136 species of shrubs and herbs with $\mathrm{H}^{\prime}$ and $\mathrm{E}$ values of 3.68 and 0.75 , respectively. The most dominant species of trees was Alstonia spatulata, of shrubs and herbs Stenochlaena palustris. The small diameter class of trees and the covering of trees with climbers indicated that the large trees had been cut. We recommend further studies with more and better-located plots to get better data on the vegetation composition and structure, including the invasion of alien species Ludwigia peruviana and the effect of climbers on trees.
\end{abstract}

Keywords: Alstonia spatulata, Camnosperma coriaceum, freshwater swamp, Ludwigia peruviana

Abbreviations: CADDB: Cagar Alam Danau Dusun Besar; TWADDTS: Taman Wisata Alam Danau Dendam Tak Sudah

\section{INTRODUCTION}

In Sumatra Island, there are three types of forested wetlands: mangrove, peat swamp forest, and freshwater swamp forest. Mangrove grows in saline substrate and is usually located in estuaries and protected shores. Peat swamp forest grows in peat, defined as a soil at least $50 \mathrm{~cm}$ deep, which contains $65 \%$ of organic matter, while freshwater swamp forest grows on soil periodically inundated with mineral-rich freshwater with $\mathrm{pH}$ of 6 or more (Whitten et al. 1984). In practice, however, the distinction between freshwater swamp forest and peat swamp forest is not always clear because the organic matter content of soil in freshwater swamp varies (Davison et al. 2018), and a few centimeters of peat may occur in freshwater swamp forest (Whitmore 1984).

In its pristine condition, freshwater swamp forest in Southeast Asia harbors a great number of plant species. In general, the flora of freshwater swamp forests does not differ at family and genus levels from that of dry land in the same region (Whitmore 1984). Even peat swamp forest which grows in nutrient-poor soil share $80 \%$ of its flora to other habitats (Giesen et al. 2018). The current literature on the freshwater swamp forest in Southeast Asia accessible online are few (Chong et al. 2018; Davison et al. 2018; Theilade et al. 2011) because it is understudied (Ho et al 2019), getting less attention than the peat swamp forests and mangrove (Clews et al. 2018). Studies on this habitat are, therefore, necessary. Another reason for this need is the fact that the Southeast Asian freshwater swamp forest is a highly threatened habitat (Ho et al 2019).

Threats to freshwater swamp forest and its surrounding dry forest in Bengkulu City have occurred extensively (Tasori et al. 2014; Eviona 2016). This freshwater swamp forest grows along the edge of a lake, named Danau Dendam Tak Sudah (meaning Unfinished Revenge Lake). The soil in the swamp is categorized as mineral soil, but it is very acidic, with $\mathrm{pH}$ of 4-5 (Sudirja and Hindersah 2009). The lake and its surrounding vegetation were designated as a strict nature reserve, called Cagar Alam Danau Dusun Besar (hereafter referred to as CADDB) with an area of 577 ha. The reason for its establishment was the presence of Papilionanthe hookeriana (Rchb.f.) Schltr (previously named Vanda hookeriana), an orchid species protected by Indonesian law (Government regulation no 7, 1999) until 2018 when the Minister of Environment and Forestry issued a new regulation which no longer listed $V$. hookeriana as a protected species (Minister of Environment and Forestry Regulation no 106, 2018). In 2019 , due to the request from the local government, part of the nature reserve area ( $88.82 \mathrm{ha}$ ) was designated as Nature Tourism Park, called Taman Wisata Alam Danau Dendam Tak Sudah (hereafter referred to as TWA DDTS), so the remaining area of nature reserve is 488.18 ha. Both strict 
nature reserve and nature tourism park, according to Indonesian laws, belong to the category of conservation forest, but more activities are allowed in the nature park than in the nature reserve (Act no 5, 1990).

This conservation forest is surrounded by settlements, so it has suffered from encroachment. Much of the forest in the swamp has been cut and the surrounding dry land has been converted into agricultural cultivation, mostly oil palm plantation (Tasori et al. 2014). A study on tree composition of the swamp forest conducted in 2007 found only small-size trees because the large ones had been cut (Permono 2007). Until this study was done, no published data on the flora of this conservation forest area were available. Therefore, this study was conducted to assess the plant species composition and diversity of conservation forest area of CADDB and TWADDTS, and to discuss the dominant species in the swamp forest.

\section{MATERIALS AND METHODS}

\section{Study area}

This study was conducted in Cagar Alam Danau Dusun Besar (CADDB) and Taman Wisata Alam Danau Dendam Tak Sudah (TWADDTS), Bengkulu, Indonesia; located at $03^{\circ} 47^{\prime} 45^{\prime}$ '-03'49'01' $\mathrm{N}$ and 102 $18^{\circ} 07^{\prime}$ '-102 $20^{\circ} 11^{\prime}$ ' E
(Figure 1). This conservation area has a wet climate with an average temperature of $29-32^{\circ} \mathrm{C}$ and relative humidity $82-87 \%$. The study site has a flat topography with slopes of $0-8 \%$, and an altitude of $15 \mathrm{~m}$. According to USDA Soil Taxonomy classification, the soil in the swamp forest is categorized as Histosol and Inceptisols, having very low fertility (Sudirja and Hindersah 2009).

\section{Data collection}

Trees with diameter at breast height (dbh) of $>10 \mathrm{~cm}$ were sampled using $10 \mathrm{~m}$ x $10 \mathrm{~m}$ plots. A total of 120 plots were located in three zones, i.e., CADDB (80 plots), rehabilitation area within CADDB (20 plots), and TWADDTS $(20$ plots $)$ The plots were placed systematically along the line located in the swamp which had many trees. The diameter and species of each tree were recorded. Because the swamp forest consisted almost exclusively of two tree species, i.e., Campnosperma coriaceum and Alstonia spatulata, we also recorded other trees that occurred only occasionally in the swamp and its edge, outside the plots, but their dbh was not measured. The outside-plot trees were assumed to have 2 individuals. Some areas of the swamp too deep to walk on were not sampled, so, there might be some species in those areas which had not been recorded.

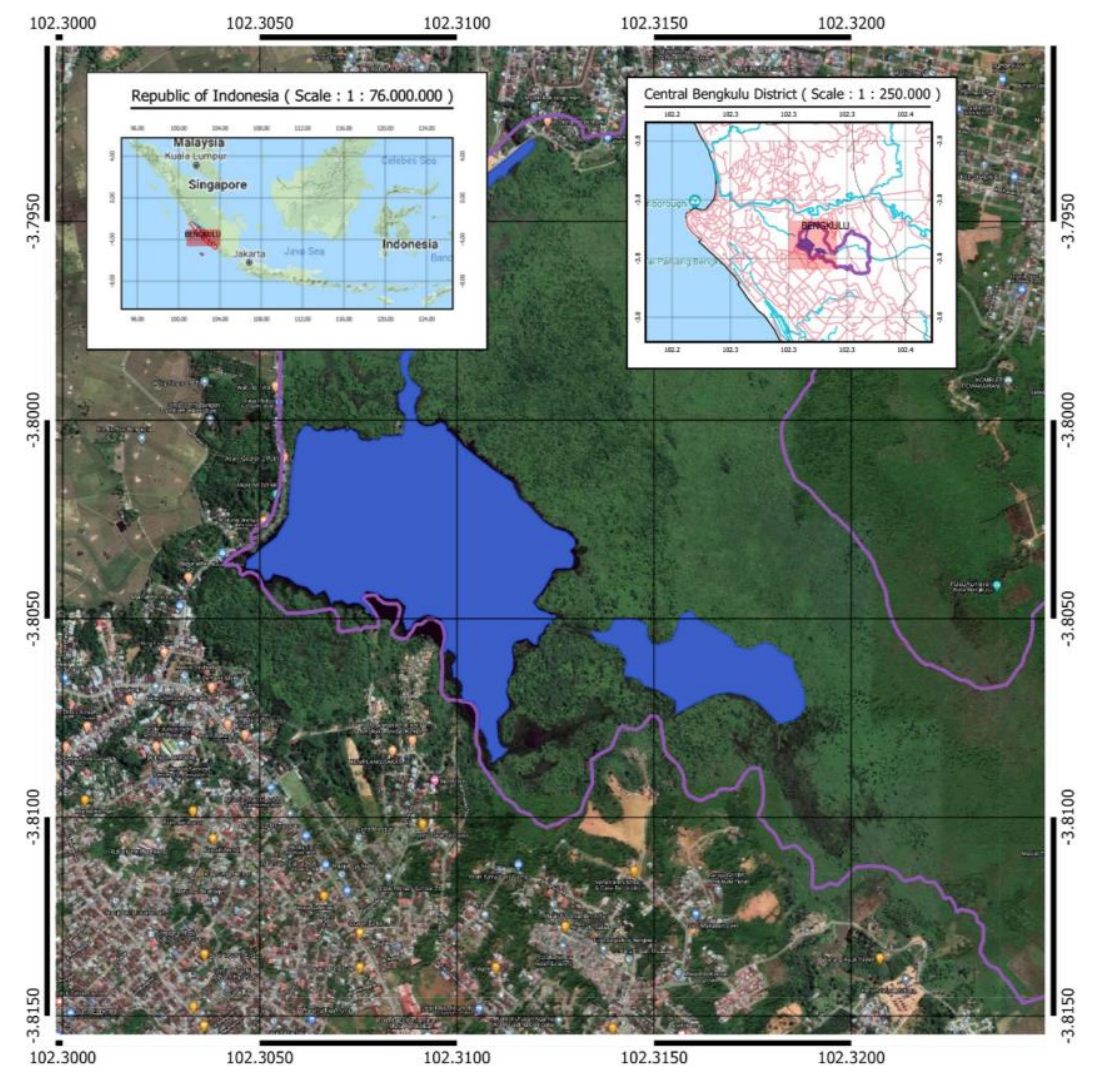

STUDY SITE:

DANAU DUSUN BESAR NATURE RESERVE \& DANAU DENDAM TAK SUDAH NATURETOURISM PARK

BENGKULU PROVINCE, INDONESIA

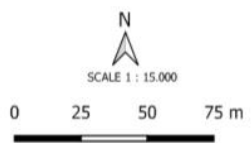

LEGENDS :

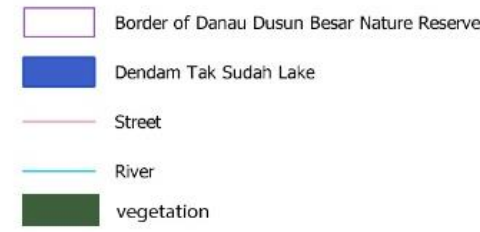

Figure 1. Location of study in Cagar Alam Danau Dusun Besar (CADDB) and Taman Wisata Alam Danau Dendam Tak Sudah (TWADDTS), Bengkulu, Indonesia 


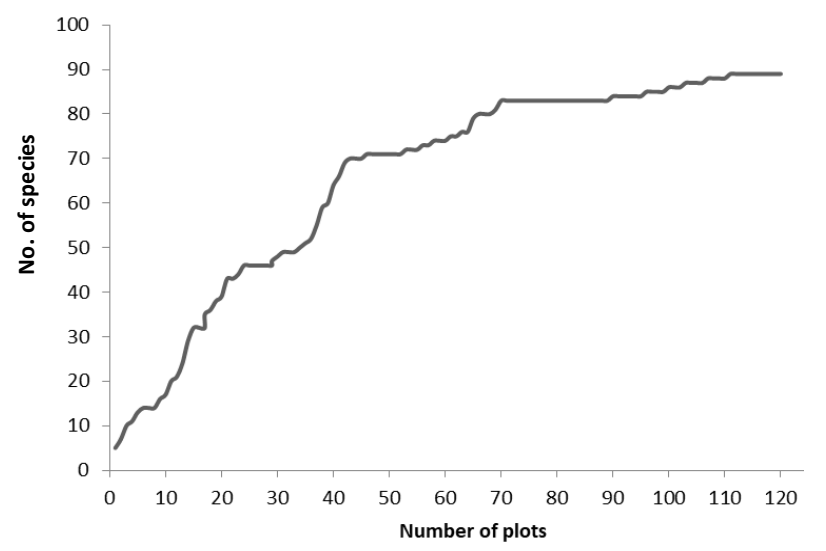

Figure 2. Species accumulating curve for shrubs and herbs in the study site, Danau Dusun Besar conservation forest, Bengkulu Province, Indonesia

Species of shrubs and herbs were sampled in 120 plots, each measuring $1 \times 1 \mathrm{~m}$, located in areas with few trees because they were found most abundant in the open areas. The cover of each species was estimated visually as a percentage. Species accumulating curve shows that the curve has flattened (Figure 2), indicating the sufficiency of the plot number. However, we also found species that occurred only occasionally outside the plots. MuellerDombois and Ellenberg (1974) say that rare species are usually not included in the plots. The outside-plot plants were recorded and the cover estimate were assumed to be same as the minimum cover estimate of the inside-lot species, i.e., $5 \%$.

Plant identification was done using identification books of Backer and van der Brink (1963), Backer and van der Brink (1965), Backer and van der Brink (1968), Soerjani et al. (1987), Noda et al. (1994 ), Moody et al. (1984), and Sukarya (2013), and websites on the internet, i.e., Flora Malesiana (http: //portal.cybertaxonomy.org/flora-malesiana), Invasive species compendium (https: //www.cabi.org/isc), Australian tropical rain forest plants (http: //www.anbg.gov.au/cpbr/cd-keys/RFK7/key/RFK7/

Media/Html/index_rfk.htm), Plants of the World online (http: //plantsoftheworldonline.org/?). Plant names followed the accepted names in the Plant List (http: //www.theplantlist.org/), except for species whose name have not been resolved, i.e., Mangifera foetida, which was taken from invasive species compendium (https: //www.cabi.org/isc/datasheet/31668), and Asplenium longissimum Johow from International Plant Names Index (https: //www.ipni.org/n/22504-2).

\section{Data analysis}

\section{Tree data}

The tree data were analyzed descriptively to show the diameter class distribution, and quantitatively to determine the Shannon-Wiener diversity index and evenness index (Mueler-Dombois and Ellenberg 1974), calculated with the following formulas:
Diversity index:

$$
\mathrm{H}^{\prime}=-\sum_{\mathrm{i}=1}^{\mathrm{s}}(\mathrm{pi})(\ln \mathrm{pi})
$$

Where:

$\mathrm{H}^{\prime}$ = species diversity index;

$\mathrm{s}=$ number of species of trees;

$\mathrm{pi}=$ proportion of the $\mathrm{i}^{\text {th }}$ species $=\mathrm{ni} / \mathrm{N}=$ (individual number of the $\mathrm{i}^{\text {th }}$ species /individual number of all species).

Evenness index:

$$
\mathrm{E}=\frac{\mathrm{H}^{\prime}}{\mathrm{LnS}}
$$

Where:

$\mathrm{E}=$ evenness index;

$\mathrm{S}=$ the number of species.

\section{Shrub and herb data}

The shrub and herb data were analyzed to determine the diversity index of Shannon-Wiener, the evenness index, and importance value (Mueller-Dombois and Ellenberg 1974). The number of individuals for the diversity index was replaced by estimate of cover. The importance value was calculated as follows:

Importance value of the $i^{\text {th }}$ Species $=$ Relative cover of the $i^{\text {th }}$ species + relative frequency of the $i^{\text {th }}$ species.

Where:

Relative cover of the $\mathrm{i}^{\text {th }}$ species $=$

$\frac{\text { Total cover of the i-th Species }}{\text { Total cover of all species }} \times 100$

Frequency of the $\mathrm{i}^{\text {th }}$ species $=$

$\frac{\text { Number of plots containing the i-th Species }}{\text { Total plots }} \times 100$

Relative frequency of the $\mathrm{i}^{\text {th }}$ species $=$

Frequency of the i-th Species $\times 100$

Note: The cover and frequency of each of the outsideplot plants were assumed to be the same as the minimum cover $(5 \%)$ and frequency $(0.83 \%)$ of the inside-plot species.

\section{RESULTS AND DISCUSSION}

\section{Species diversity and composition of trees}

The number of tree species in the study site was 23 (Table 1), belonging to 10 families, with Shannon-Wiener diversity index of 0.29 , and evenness index of 0.09 . Only two species of trees were found within the plots, namely Alstonia spatulata Blume and Campnosperma corieaceum (Jack) Hallier f. Other species of trees were found occasionally outside the plots, mostly at the edge of the swamp. Both Alstonia and Campnosperma had the highest number of individuals in 15.0-19.9 $\mathrm{cm}$ diameter class (Figure 3). The biggest diameter of Alstonia tree was in the 
30-34.9 cm diameter class, and Campnosperma 25.0-29.9 cm diameter class.

\section{Species diversity and composition of shrubs and herbs}

The number of identified shrub and herb species was 136, belonging to 51 families (Table 2), with the ShannonWiener diversity index of 3. 58 and the evenness index 0.75 . The top four families with the highest number of species were Fabaceae (17), Asteraceae (14), Poaceae (13), and Cyperaceae (12). Each of other families had less than 10 species. In addition, 7 unidentified species were also found.

Five species that had importance value higher than 10 were Stenochlaena palustris (Burm. f.) Bedd., Scleria ciliaris Nees, Ludwigia peruviana (L.) H.Hara, Persicaria attenuata (R. Br.) Soják, and Actinoscirpus grossus (L.f.) Goetgh. \& D.A. Simpson. Based on the life forms, the shrubs and herbs could arbitrarily be divided into forb which was 58 species, shrub 34, fern 18, grass 13, sedge 9, and liana 4 (Table 2).

\section{Palm}

In addition to tree and ground cover species, two species of palm were found in the swamp and adjacent dry land within the conservation forest, namely Nypa fruticans Wurmb and Elaeis guineensis Jacq.

\section{Discussion}

Trees

The diversity index $\left(\mathrm{H}^{\prime}\right)$ of tree species in the study site was only 0.29 , considered very low, presumably due to the low species evenness (0.09) and low species richness (only 23). In comparison, in a fresh swamp forest of Cagar Alam Rimbo Panti West Sumatra, Yusuf and Purwaningsih (2009) found 136 species of trees with $\mathrm{dbh}>10 \mathrm{~cm}$ from 40 families. Meanwhile, Chong et al. (2018) found 288 species of trees with $\mathrm{dbh}>5 \mathrm{~cm}$ from 60 families in a freshwater swamp forest of Singapore. The low tree diversity in the study site was presumably caused by illegal cutting and forest encroachment (Tasori et al. 2014; Eviona 2016).

The majority of tree species recorded in this study were found in the edge of the swamp where the soil is not permanently inundated. The inundated area was dominated by Alstonia spatulata and Campnosperma coriaceum. Alstonia spatulata, is locally called pulai gabus, the Indonesian word for cork, referring to its ultralight root wood and basalmost stemwood which has been traditionally used as replacement of cork (Baas and Fujii 2020). It is a native species that naturally grows in Burma (Myanmar), Thailand, Cambodia, Vietnam, Peninsular Malaysia, Singapore, Sumatra, Bangka, Java, Borneo, and New Guinea (Slik 2009 onwards). This species was dominant in the study site because it can grow in poorly drained and frequently flooded soil (Teo 2001). In ancient swamp forest bordering a lake in Bantam (Banten), Endert in 1932 found $A$. spatulata one of the dominant species (cited in Backer and Van der Brink 1965). The genus
Alstonia in general is commonly found in swamp forests in Southeast Asia and New Guniea (Whitmore 1984).

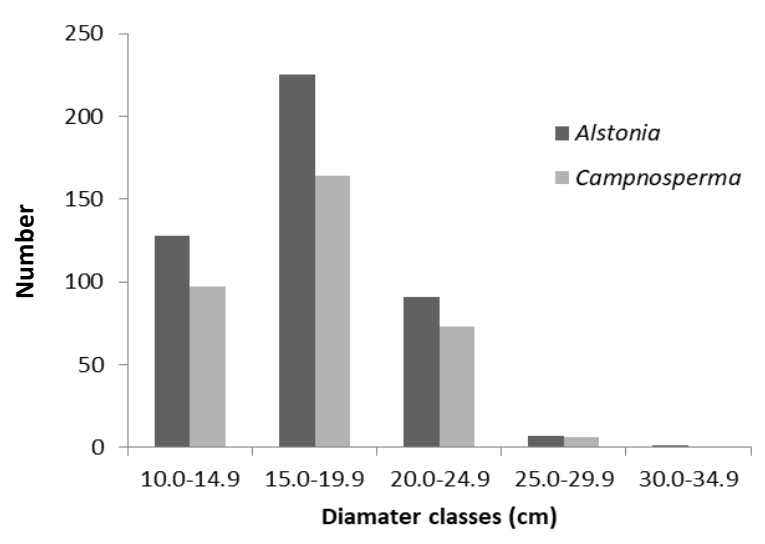

Figure 3. Diameter distribution of Alstonia spatulata and Campnosperma coriaceum

Table 1. List of tree species in the study site, , Danau Dusun Besar conservation forest, Bengkulu Province, Indonesia

\begin{tabular}{|c|c|c|}
\hline Species & Family & $\begin{array}{c}\text { No. of } \\
\text { individuals }\end{array}$ \\
\hline Alstonia spatula Blume & Apocynaceae & 452 \\
\hline $\begin{array}{l}\text { Campnosperma coriaceum (Jack) } \\
\text { Hallier f. }\end{array}$ & Anacardiaceae & 340 \\
\hline Lannea coromandelica (Houtt.) Merr. & Anacardiaceae & 2 \\
\hline Mangifera foetida Lour. & Anacardiaceae & 2 \\
\hline Cerbera manghas $\mathrm{L}$. & Apocynaceae & 2 \\
\hline Trema orientalis (L.) Blume & Cannabaceae & 2 \\
\hline Terminalia catappa $\mathrm{L}$. & Combretaceae & 2 \\
\hline $\begin{array}{l}\text { Hevea brasiliensis (Willd. ex } \\
\text { A.Juss.) Müll.Arg. }\end{array}$ & Euphorbiaceae & 2 \\
\hline Macaranga sp. & Euphorbiaceae & 2 \\
\hline $\begin{array}{l}\text { Mallotus paniculatus (Lam.) } \\
\text { Müll.Arg. }\end{array}$ & Euphorbiaceae & 2 \\
\hline Acacia mangium Willd. & Fabaceae & 2 \\
\hline $\begin{array}{l}\text { Archidendron pauciflorum (Benth.) } \\
\text { I.C.Nielsen }\end{array}$ & Fabaceae & 2 \\
\hline Gliricidia sepium (Jacq.) Walp. & Fabaceae & 2 \\
\hline Pterocarpus indicus Willd. & Fabaceae & 2 \\
\hline Peronema canescens Jack & Lamiaceae & 2 \\
\hline Tectona grandis L.f. & Lamiaceae & 2 \\
\hline Vitex pinnata $\mathrm{L}$ & Lamiaceae & 2 \\
\hline $\begin{array}{l}\text { Cinnamomum porrectum (Roxb.) } \\
\text { Kosterm. }\end{array}$ & Lauraceae & 2 \\
\hline Ficus benjamina $\mathrm{L}$. & Moraceae & 2 \\
\hline Ficus fistulosa Reinw. ex Blume & Moraceae & 2 \\
\hline Ficus septica Burm.f. & Moraceae & 2 \\
\hline Psidium guajava $\mathrm{L}$ & Myrtaceae & 2 \\
\hline Syzygium sp. & Myrtaceae & 2 \\
\hline
\end{tabular}

Note: The number of individuals of species other than Alstonia and Campnosperma was assumed to be 2 
Table 2. The list of species shrubs and herbs with their importance values, families and life forms in the study site

\begin{tabular}{|c|c|c|c|c|c|}
\hline Species & Family & Life form & $\begin{array}{l}\text { Rel. } \\
\text { cover }\end{array}$ & $\begin{array}{l}\text { Rel. } \\
\text { freq. }\end{array}$ & IV \\
\hline Stenochlaena palustris (Burm. f.) Bedd. & Blechnaceae & Climbing fern & 10.51 & 7.74 & 18.25 \\
\hline Scleria ciliaris Nees & Cyperaceae & Sedge & 7.04 & 7.74 & 14.78 \\
\hline Ludwigia peruviana (L.) H.Hara & Onagraceae & Shrub & 8.49 & 5.23 & 13.72 \\
\hline Persicaria attenuata (R. Br.) Soják & Polygalaceae & Erect forb & 5.66 & 5.65 & 11.31 \\
\hline Actinoscirpus grossus (L.f.) Goetgh. \& D.A.Simpson. & Cyperaceae & Sedge & 6.61 & 3.97 & 10.58 \\
\hline Crinum asiaticum $\mathrm{L}$. & Amaryllidaceae & Erect forb & 7.29 & 2.93 & 10.22 \\
\hline Nepenthes mirabilis (Lour.) Druce & Nepentheaceae & Climbing forb & 3.60 & 3.14 & 6.74 \\
\hline Uncaria acida (Hunter) Roxb & Rubiaceae & Liana & 3.09 & 3.56 & 6.65 \\
\hline Mimosa pigra $\mathrm{L}$. & Fabaceae & Shrub & 3.00 & 3.56 & 6.56 \\
\hline Mikania micrantha Kunth & Asteraceae & Climbing forb & 1.97 & 3.14 & 5.11 \\
\hline Colocasia esculenta (L.) Schott & Araceae & Erect forb & 2.57 & 2.51 & 5.08 \\
\hline Cyclosorus interruptus (Willd.) H. Itô & Thelypteridaceae & Terrestrial fern & 2.10 & 2.72 & 4.82 \\
\hline Imperata cylindrica (L.) Raeusch. & Poaceae & Grass & 1.97 & 2.09 & 4.07 \\
\hline Panicum maximum Jacq. & Poaceae & Creeping forb & 2.02 & 1.67 & 3.69 \\
\hline Merremia umbellata (L.) Hallier f & Convolvulaceae & Climbing forb & 1.63 & 1.67 & 3.30 \\
\hline Paspalum distichum $\mathrm{L}$. & Poaceae & Spreading shrub & 1.54 & 1.26 & 2.80 \\
\hline Fimbristylis littoralis Gaudich. & Cyperaceae & Sedge & 1.72 & 1.05 & 2.76 \\
\hline Ludwigia octovalvis (Jacq.) P.H.Raven & Onagraceae & Shrub & 1.72 & 1.05 & 2.76 \\
\hline Asystasia gangetica (L.) T.Anderson & Acanthaceae & Creeping forb & 0.94 & 1.46 & 2.41 \\
\hline Ludwigia hissopifolia (G.Don) Exell & Onagraceae & Shrub & 1.50 & 0.84 & 2.34 \\
\hline Senna alata (L.) Roxb & Fabaceae & Shrub & 1.20 & 1.05 & 2.25 \\
\hline Dicranopteris linearis (Burm. f.) Underw. & Gleicheniaceae & Terrestrial fern & 0.94 & 1.26 & 2.20 \\
\hline Ipomoea aquatica Forssk. & Convolvulaceae & Creeping forb & 1.54 & 0.63 & 2.17 \\
\hline Nephrolepis biserrata (Sw.) Schott & Nephrolepidaceae & Terrestrial fern & 0.69 & 1.26 & 1.94 \\
\hline Davallia denticulata (Burm. f.) Mett. ex Kuhn & Davalliaceae & Epiphytic or terrestrial fern & 0.86 & 1.05 & 1.90 \\
\hline Melastoma malabathricum $\mathrm{L}$. & Mellastomaceae & Shrub & 0.86 & 0.84 & 1.69 \\
\hline Chamaecrista mimosoides (L.) Greene. & Fabaceae & Shrub & 1.03 & 0.42 & 1.45 \\
\hline Psychotria sarmentosa Blume & Rubiaceae & Liana & 0.60 & 0.84 & 1.44 \\
\hline Sphagneticola trilobata (L.) Pruski & Asteraceae & Creeping forb & 0.94 & 0.42 & 1.36 \\
\hline Asplenium longissimum Johow & Aspleniaceae & Terrestrial fern & 0.47 & 0.84 & 1.31 \\
\hline Thunbergia alata Bojer ex Sims & Acanthaceae & Climbing forb & 0.47 & 0.84 & 1.31 \\
\hline Pteris vittata $\mathrm{L}$ & Pteridaceae & Terrestrial fern & 0.51 & 0.63 & 1.14 \\
\hline Centrosema molle Benth. & Fabaceae & Climbing forb & 0.43 & 0.63 & 1.06 \\
\hline Mimosa invisa Colla & Fabaceae & Climbing herb & 0.51 & 0.42 & 0.93 \\
\hline Salvinia adnata Desv. & Salviniaceae & Aquatic fern & 0.51 & 0.42 & 0.93 \\
\hline Schoenoplectiella mucronata (L.) J.Jung \& H.K.Choi & Cyperaceae & Sedge & 0.30 & 0.63 & 0.93 \\
\hline Blumea balsamifera (L.) DC. & Asteraceae & Shrub & 0.43 & 0.42 & 0.85 \\
\hline Ludwigia adscendens (L.) H.Hara & Onagraceae & Creeping forb & 0.43 & 0.42 & 0.85 \\
\hline Lycopodiella cernua (L.) Pic. Serm. & Lycopodiaceae & Creeping fern & 0.43 & 0.42 & 0.85 \\
\hline Waltheria indica $\mathrm{L}$. & Malvaceae & Shrub & 0.60 & 0.21 & 0.81 \\
\hline Ardisia humilis Vahl & Rubiaceae & Shrub & 0.34 & 0.42 & 0.76 \\
\hline Manihot esculenta Crantz & Euphorbiaceae & Shrub & 0.34 & 0.42 & 0.76 \\
\hline Mimosa pudica L. & Fabaceae & Creeping forb & 0.34 & 0.42 & 0.76 \\
\hline Polygala paniculata $\mathrm{L}$ & Polygalaceae & Erect forb & 0.34 & 0.42 & 0.76 \\
\hline Echinochloa colona (L.) Link & Poaceae & Grass & 0.26 & 0.42 & 0.68 \\
\hline Flagellaria indica $\mathrm{L}$. & Flagellariaceae & Semi woody climber & 0.26 & 0.42 & 0.68 \\
\hline Nepenthes ampularia Jack & Nepenthaceae & Climbing forb & 0.26 & 0.42 & 0.68 \\
\hline Nephrolepis cordifolia (L.) C. Presl & Nephrolepidaceae & Terrestrial fern & 0.26 & 0.42 & 0.68 \\
\hline $\begin{array}{l}\text { Phymatosorus pustulatus (G. Forst.) Large, Braggins \& } \\
\text { P.S. Green }\end{array}$ & Polypodiaceae & Terrestrial or epiphytic fern & 0.26 & 0.42 & 0.68 \\
\hline Chromolaena odorata (L.) R.M.King \& H.Rob. & Asteraceae & Climbing ferb, woody at old age & 0.17 & 0.42 & 0.59 \\
\hline Chrysopogon aciculatus (Retz.) Trin. & Poaceae & Grass & 0.17 & 0.42 & 0.59 \\
\hline Elephantopus tomentosus L. & Asteraceae & Grass & 0.17 & 0.42 & 0.59 \\
\hline Fimbristylis aestivalis Vahl & Cyperaceae & Sedge & 0.17 & 0.42 & 0.59 \\
\hline Ipomoea triloba $\mathrm{L}$. & Convolvulaceae & Creeping forb & 0.17 & 0.42 & 0.59 \\
\hline Lygodium longifolium (Willd.) Sw & Lygodiaceae & Climbing fern & 0.17 & 0.42 & 0.59 \\
\hline Rhynchospora corymbosa (L.) Britton & Cyperaceae & Sedge & 0.17 & 0.42 & 0.59 \\
\hline Alpinia zerumbet (Pers.) B.L.Burtt \& R.M.Sm & Zingiberacea & Erect forb & 0.34 & 0.21 & 0.55 \\
\hline Cleome rutidosperma DC. & Cleomaceae & Spreading to erect forb & 0.34 & 0.21 & 0.55 \\
\hline Axonopus compressus (Sw.) P.Beauv. & Poaceae & Grass & 0.26 & 0.21 & 0.47 \\
\hline Breynia vitis-idaea (Burm.f.) C.E.C.Fisch. & Phyllantaceae & Shrub & 0.26 & 0.21 & 0.47 \\
\hline Celosia argentea $\mathrm{L}$. & Amaranthaceae & Forb & 0.26 & 0.21 & 0.47 \\
\hline
\end{tabular}


Hyptis capitata Jacq.

Hyptis suaveolens (L.) Poit.

Ipomoea fistulosa Mart. ex Choisy

Kyllinga polyphylla Willd. ex Kunth

Oxalis corniculata $\mathrm{L}$

Aeschynanthus radicans Jack

Alysicarpus vaginalis (L.) DC.

Calopogonium mucunoides Desv.

Crotalaria pallida Aiton

Dactyloctenium aegyptium (L.) Willd.

Sida acuta Burm.f

Sida rhombifolia $\mathrm{L}$.

Stachytarpheta jamaicensis (L.) Vahl

Artanema longifolium (L.) Vatke

Ceratopteris thalictroides (L.) Brongn.

Eclipta prostrata (L.) L.

Fuirena umbellata Rottb.

Hedyotis corymbosa (L.) Lam.

Limnocharis flava (L.) Buchenau

Lygodium microphyllum (Cav.) R. Br.

Melochia corchorifolia $\mathrm{L}$.

Oxalis barrelieri L

Parietaria judaica $L$

Pennisetum purpureum Schumach

Peperomia pellucida (L.) Kunth

Phyllanthus niruri L.

Stylosanthes humilis Kunth

Croton hirtus L'Hér

Acalypha indica L.*

Ageratum conyzoides (L.) L.

Asplenium nidus $L$.

Brucea javanica (L.) Merr.

Calotropis gigantea (L.) Dryand.

Cenchrus echinatus L.

Cleistanthus monoicus (Lour.) Müll.Arg.

Clibadium surinamense $\mathrm{L}$.

Clidemia hirta (L.) D. Don

Clitoria laurifolia Poir.

Crassocephalum crepidioides (Benth.) S.Moore

Cyanthillium cinereum (L.) H. Rob.

Cymbopogon calcicola C.E.Hubb.

Cynodon dactylon (L.) Pers.

Cyperus procerus Rottb.

Cyperus rotundus $\mathrm{L}$.

Desmodium heterophyllum (Willd.) DC.

Dioscorea hispida Dennst.

Diplazium esculentum (Retz.) Sw

Eleusine indica (L.) Gaertn.

Eragrostis amabilis (L.) Wight \& Arn.

Euphorbia heterophylla L.

Euphorbia hirta L.

Lantana camara L.

Leucas zeylanica (L.) W.T.Aiton

Lindsaea ensifolia Sw.

Ludwigia perennis $\mathrm{L}$.

Musa paradisiaca $L$

Mussaenda frondosa $\mathrm{L}$.

Nymphaea lotus L.

Oldenlandia auricularia (L.) K.Schum

Papilionanthe hookeriana (Rchb.f.) Schltr.

Passiflora foetida $\mathrm{L}$.

Pericampylus glaucus (Lam.) Merr.

Phyllanthus amarus Schumach. \& Thonn.

Phyllanthus urinaria $\mathrm{L}$.

Phyllanthus virgatus G.Forst.

Phymatosorus scolopendria (Burm. f.) Pic. Serm

Plectranthus amboinicus (Lour.) Spreng.

\begin{tabular}{|c|c|}
\hline Lamiaceae & Shrub \\
\hline Lamiaceae & Shrub \\
\hline Convolvulaceae & Creeping shrub \\
\hline Cyperaceae & Sedge \\
\hline Oxalidaceae & Erect forb \\
\hline Gesneriaceae & Climbing forb \\
\hline Fabaceae & Creeping forb \\
\hline Fabaceae & Shrub \\
\hline Fabaceae & Erect forb \\
\hline Poaceae & Grass \\
\hline Malvaceae & Shrub \\
\hline Malvaceae & Shrub \\
\hline Verbenaceae & Shrub \\
\hline Linderniaceae & Erect forb \\
\hline Pteridaceae & Aquatic fern \\
\hline Asteraceae & Prostating forb \\
\hline Cyperaceae & Sedge \\
\hline Rubiaceae & Erect forb \\
\hline Alismataceae & Floating forb \\
\hline Lygodiaceae & Climbing fern \\
\hline Malvaceae & Shrub \\
\hline Oxalidaceae & Erect forb \\
\hline Urticaceae & Erect or prostating forb \\
\hline Poaceae & Grass \\
\hline Piperaceae & Grass \\
\hline Phyllantaceae & Erect forb \\
\hline Fabaceae & Creeping forb \\
\hline Euphorbiacea & Erect forb \\
\hline Euphorbiaceae & Erect forb \\
\hline Asteraceae & Erect forb \\
\hline Aspleniaceae & Epiphytic and terrestrial fern \\
\hline Simaroubaceae & Shrub \\
\hline Apocynaceae & Shrub \\
\hline Poaceae & Grass \\
\hline Phyllantaceae & Shrub \\
\hline Asteraceae & Shrub \\
\hline Melastomaceae & Shrub \\
\hline Fabaceae & Shrub \\
\hline Asteraceae & Erect forb \\
\hline Asteraceae & Erect forb \\
\hline Poaceae & Grass \\
\hline Poaceae & Grass \\
\hline Cyperaceae & Sedge \\
\hline Cyperaceae & Sedge \\
\hline Fabaceae & Creeping forb \\
\hline Dioscoreaceae & Climbing forb \\
\hline Athryiaceae & Terrestrial fern \\
\hline Poaceae & Grass \\
\hline Poaceae & Grass \\
\hline Euphorbiaceae & Erect forb \\
\hline Euphorbiaceae & Erect forb \\
\hline Verbenaceae & Shrub \\
\hline Lamiaceae & Erect forb \\
\hline Lindsaeaceae & Epiphytic or terrestrial fern \\
\hline Onagraceae & Shrub \\
\hline Musaceae & Large forb \\
\hline Rubiaceae & Shrub \\
\hline Nymphaeaceae & Aquatic forb \\
\hline Rubiaceae & Erect forb \\
\hline Orchidaceae & Climbing forb \\
\hline Passifloraceae & Climbing-creeping forb \\
\hline Menispermaceae & Liana \\
\hline Phyllantaceae & Shrub \\
\hline Phyllantaceae & Erect forb \\
\hline Phyllantaceae & Erect forb \\
\hline Polypodiaceae & Terrestrial fern \\
\hline Lamiaceae & Erect forb \\
\hline
\end{tabular}

0.26

$\begin{array}{lll}0.26 & 0.21 & 0.47\end{array}$

$\begin{array}{lll}0.26 & 0.21 & 0.47\end{array}$

$\begin{array}{lll}0.26 & 0.21 & 0.47\end{array}$

$\begin{array}{lll}0.26 & 0.21 & 0.47\end{array}$

$\begin{array}{lll}0.17 & 0.21 & 0.38\end{array}$

$\begin{array}{lll}0.17 & 0.21 & 0.38\end{array}$

$\begin{array}{lll}0.17 & 0.21 & 0.38\end{array}$

$\begin{array}{lll}0.17 & 0.21 & 0.38\end{array}$

$\begin{array}{lll}0.17 & 0.21 & 0.38\end{array}$

$\begin{array}{lll}0.17 & 0.21 & 0.38\end{array}$

$\begin{array}{lll}0.17 & 0.21 & 0.38\end{array}$

$\begin{array}{lll}0.17 & 0.21 & 0.38\end{array}$

$\begin{array}{lll}0.09 & 0.21 & 0.30\end{array}$

$\begin{array}{lll}0.09 & 0.21 & 0.30\end{array}$

$\begin{array}{lll}0.09 & 0.21 & 0.30\end{array}$

$\begin{array}{lll}0.09 & 0.21 & 0.30\end{array}$

$\begin{array}{lll}0.09 & 0.21 & 0.30\end{array}$

$\begin{array}{lll}0.09 & 0.21 & 0.30\end{array}$

$\begin{array}{lll}0.09 & 0.21 & 0.30\end{array}$

$\begin{array}{lll}0.09 & 0.21 & 0.30\end{array}$

$\begin{array}{lll}0.09 & 0.21 & 0.30\end{array}$

$\begin{array}{lll}0.09 & 0.21 & 0.30\end{array}$

$\begin{array}{lll}0.09 & 0.21 & 0.30\end{array}$

$\begin{array}{lll}0.09 & 0.21 & 0.30\end{array}$

$\begin{array}{lll}0.09 & 0.21 & 0.30\end{array}$

$\begin{array}{lll}0.09 & 0.21 & 0.30\end{array}$

$\begin{array}{lll}0.04 & 0.21 & 0.25\end{array}$

$\begin{array}{lll}0.04 & 0.21 & 0.25\end{array}$

$\begin{array}{lll}0.04 & 0.21 & 0.25\end{array}$

$\begin{array}{lll}0.04 & 0.21 & 0.25\end{array}$

$\begin{array}{lll}0.04 & 0.21 & 0.25\end{array}$

$\begin{array}{lll}0.04 & 0.21 & 0.25\end{array}$

$\begin{array}{lll}0.04 & 0.21 & 0.25\end{array}$

$\begin{array}{lll}0.04 & 0.21 & 0.25\end{array}$

$\begin{array}{lll}0.04 & 0.21 & 0.25\end{array}$

$\begin{array}{lll}0.04 & 0.21 & 0.25\end{array}$

$\begin{array}{lll}0.04 & 0.21 & 0.25\end{array}$

$\begin{array}{lll}0.04 & 0.21 & 0.25\end{array}$

$\begin{array}{lll}0.04 & 0.21 & 0.25\end{array}$

$\begin{array}{lll}0.04 & 0.21 & 0.25\end{array}$

$\begin{array}{lll}0.04 & 0.21 & 0.25\end{array}$

$\begin{array}{lll}0.04 & 0.21 & 0.25\end{array}$

$\begin{array}{lll}0.04 & 0.21 & 0.25\end{array}$

$\begin{array}{lll}0.04 & 0.21 & 0.25\end{array}$

$\begin{array}{lll}0.04 & 0.21 & 0.25\end{array}$

$\begin{array}{lll}0.04 & 0.21 & 0.25\end{array}$

$\begin{array}{lll}0.04 & 0.21 & 0.25\end{array}$

$\begin{array}{lll}0.04 & 0.21 & 0.25\end{array}$

$\begin{array}{lll}0.04 & 0.21 & 0.25\end{array}$

$\begin{array}{lll}0.04 & 0.21 & 0.25\end{array}$

$\begin{array}{lll}0.04 & 0.21 & 0.25\end{array}$

$\begin{array}{lll}0.04 & 0.21 & 0.25\end{array}$

$\begin{array}{lll}0.04 & 0.21 & 0.25\end{array}$

$\begin{array}{lll}0.04 & 0.21 & 0.25\end{array}$

$\begin{array}{lll}0.04 & 0.21 & 0.25\end{array}$

$\begin{array}{lll}0.04 & 0.21 & 0.25\end{array}$

$\begin{array}{lll}0.04 & 0.21 & 0.25\end{array}$

$\begin{array}{lll}0.04 & 0.21 & 0.25\end{array}$

$\begin{array}{lll}0.04 & 0.21 & 0.25\end{array}$

$\begin{array}{lll}0.04 & 0.21 & 0.25\end{array}$

$\begin{array}{lll}0.04 & 0.21 & 0.25\end{array}$

$\begin{array}{lll}0.04 & 0.21 & 0.25\end{array}$

$\begin{array}{lll}0.04 & 0.21 & 0.25\end{array}$

$\begin{array}{lll}0.04 & 0.21 & 0.25\end{array}$

$\begin{array}{lll}0.04 & 0.21 & 0.25\end{array}$

$\begin{array}{lll}0.04 & 0.21 & 0.25\end{array}$ 
Premna serratifolia $\mathrm{L}$ Richardia brasiliensis Gomes Senna tora (L.) Roxb. Sesbania sesban (L.) Merr. Spermacoce laevis Lam. Synedrella nodiflora (L.) Gaertn. Tetracera sarmentosa (L.) Vahl Tridax procumbens (L.) L. Total Species 1 Species 2 Species 3 Species 4 Species 5 Species 6 Species 7

\begin{tabular}{|c|c|c|c|c|}
\hline Lamiaceae & Shrub & 0.04 & 0.21 & 0.25 \\
\hline Rubiaceae & Creeping forb & 0.04 & 0.21 & 0.25 \\
\hline Fabaceae & Shrub & 0.04 & 0.21 & 0.25 \\
\hline Fabaceae & Shrub & 0.04 & 0.21 & 0.25 \\
\hline Rubiaceae & Erect forb & 0.04 & 0.21 & 0.25 \\
\hline Asteraceae & Erect forb & 0.04 & 0.21 & 0.25 \\
\hline Dilleniaceae & Liana & 0.04 & 0.21 & 0.25 \\
\hline \multirow[t]{9}{*}{ Asteraceae } & Creeping forb & 0.04 & 0.21 & 0.25 \\
\hline & & 100.00 & 100.00 & 200.00 \\
\hline & liana & & & \\
\hline & Shrub & & & \\
\hline & Liana & & & \\
\hline & Shrub & & & \\
\hline & terrestrial fern & & & \\
\hline & Liana & & & \\
\hline & Climbing herb & & & \\
\hline
\end{tabular}

*Note: IV = importance value. The species from Acalypha downward were found outside the plots.

Another frequently found tree species in the swamp was Campnosperma coriaceum, also called terentang paya. This species is naturally found in Peninsular Thailand, Peninsular Malaysia, Sumatra, Borneo and New Guinea (Slik 2009 onward). It usually grows in swamp forests in Southeast Asia and New Guniea (Whitmore 1984), but its distribution is not limited to swamp, because it is also found in other types of ecosystems (Giesen et al. 2018).

Both Alstonia and Campnosperma had small diameter. The biggest proportion of trees had a diameter class of 15.0-19,9 cm. Campnosperma even had the biggest diameter smaller than $30 \mathrm{~cm}$, while Alstonia had only two individuals with a diameter class of $30.0-34.9 \mathrm{~cm}$. In undisturbed forest, Campnosperma coriaceum may have a diameter of $90 \mathrm{~cm}$, while Alstonia spatulata $40 \mathrm{~cm}$ (Flora Malesiana 2020). The small size of trees, in addition to the low diversity, indicates that the swamp forest has been cleared. Another indication of forest clearing was the abundance of climbers, which usually thrive in young secondary forests (Paul and Yavitt 2011). The crowns of Alstonia and Campnosperma were almost invisible because they were covered by climbers (Figure 4).

\section{Shrubs and herbs}

The species richness of shrubs and herbs was 136, with the Shannon-Wiener diversity index of 3.68, considered high because the value of $\mathrm{H}^{\prime}$ in most studies falls between 1.5 and 3.5 (Margurran 2004). In addition, 7 unidentified was also found. Considering that many species were found outside the plots, there might be many other species that were not recorded in this study. Mueller-Dombois and Ellenberg (1974) state that species that occur only occasionally may not be included in the plots.

Among the shrubs and herbs, Stenochlaena palustris had the highest importance value, i.e., 18.75 (Table 2). This species is a long scrambling fern commonly found in swampy places, but it also grows in dry lands, such as open places, secondary forests, rubber gardens, oil palm plantations, river banks, and roadsides (Chai 2016). In 1938, Van Steenis reported that plenty of S.palustris was found in swamp forests in Tanjung Priuk, Jakarta (cited in Backer and Van der Brink 1965). This species is widely distributed, including tropical Asia, northeast Australia, and eastwards to Tonga and Samoa (Flora Malesiana 2020). In Sarawak, Malaysia, this edible fern is sold in restaurants (Chai 2016). In the study site, S.palustris was not only very abundant on the ground but also in the forest canopy, because this species climbed up the tree and covered the tree crowns of Alstonia and Campnosperma almost completely.

The next abundant climber in the swamp was Nepenthes mirabilis with an importance value of 6.74 , the $7^{\text {th }}$ highest among the ground cover in this study site (Table 2 ). It is not surprising that $N$. mirabilis was abundant in this study site, which has very low soil fertility (Sudirja and Hindersah 2009), because the genus Nepenthes, in general, can grow in nutrient-poor soil (Adam et al. 1992). In two study sites in South Sumatra which had very acidic soil with low nutrient content, $N$. mirabilis was the only species of Nepenthes found (Mardhiana et al. 2012). Among the species of Nepenthes, this species was the most widely distributed, stretching from Thailand, Indo-China, Peninsular Malaysia, through Indonesia, the Philippines, New Guinea to Australia, and this species usually grows abundantly in swamp (Adam et al. 1992). In Bangka Belitung Province, N. mirabiilis was also found in swamp forest (Rizqiani et al. 2018), and in peat swamp of Borneo, N.mirabilis was also the dominant climber (Hidayat et al. 2018). In Halmahera Island, N. mirabilis was found in lowland forest and edge of swamp (Cahyono et al. 2019).

Another abundant climber was Uncaria acida with an importance value of 6.65 , ranked $7^{\text {th }}$ among ground cover species (Table 2). Like other species in the genus Uncaria, this liana has hooks on its stem, enabling it to climb trees. This species can be locally abundant in humid primary and secondary forest, also along rivers, and swampy areas, at low altitudes (Backer and Van der Brink 1965; Subarnas 2001). This species was previously considered extinct in Singapore, but it has been found twice recently (Turner 2018). In peat swamp forest in Berbak National Park in Jambi Province, Sumatra $U$. acida survived forest fire occurring in the peat swamp (Eik et al. 2009). Another Uncaria, i.e., U. longifolia was found in freshwater swamp in Singapore (Tan et al 2013). 
Among the shrubs, Ludwigia peruviana was the most abundant in the study site, with an importance value of 13.72 , ranked $3^{\text {rd }}$ among the ground cover (Table 2; Figure 4). This species, also known as Peruvian primrose, Peruvian water primrose, ludwigia, Peruvian primrose bush, water primrose, is native to Mexico, Central America, the Caribbean, and South America, but has been naturalized in tropical and sub-tropical areas in the world and regarded as an environmental weed in Australia since 1971. Within 25 years, it occupied $40 \%$ of wetlands in Australia (Chandrasena et al. 2002). This species which usually grows in wetland has become a serious threat to biodiversity in Dhansiri and Kopili catchment areas of Assam, North East India because it has replaced the native species, even forming pure stand (Barua et al. 2017). This species was also a threat to restoration of broadleaf marshes on the Kissimmee floodplain, in Central Florida, USA (Toth 2010). In Sri Lanka, infestations of Ludwigia peruviana and L. octovalvis have increased in reclaimed marshlands and drainage canals, while several other species of Ludwigia continue to be major weed problems in rice agriculture (Rajapakse et al. 2011). In Indonesia, L. peruviana is considered weed of minor importance to rice
(Soerjani et al. 1987). In Pantnagar, India, another species from the same genus, i.e., L. perennis is an invasive alien species threatening the native species. Due to similarity in climate, exotic plants from tropical American rank the most dominant invasive alien species in Pantnagar (Rastogi et al. 2015).

Growing in mixed stands with $S$. palustris and $L$. peruviana was Scleria ciliaris, a species of Cyperaceae. It had an importance value of 14.78 , ranked $2^{\text {nd }}$ among the ground cover species (Table 2; Figure 5). This species is native to tropical Asia, tropical Australia, the Solomons, and Carolines (Flora Malesiana 2020). In a rubber plantation near Bukit Dua Belas National Park in Jambi, it became the second most dominant invasive species (Wahyuni et al. 2015). Meanwhile, in grassland dominated by Imperata cylindrica in Katingan District, Central Kalimantan Province, S.ciliaris was also the second dominant species (Usmadi et al. 2020). S. bancana (synonym of S. ciliaris) was also found in coalmine overburden dumping site in Satui, South Kalimantan Province (Novianti et al. 2017).

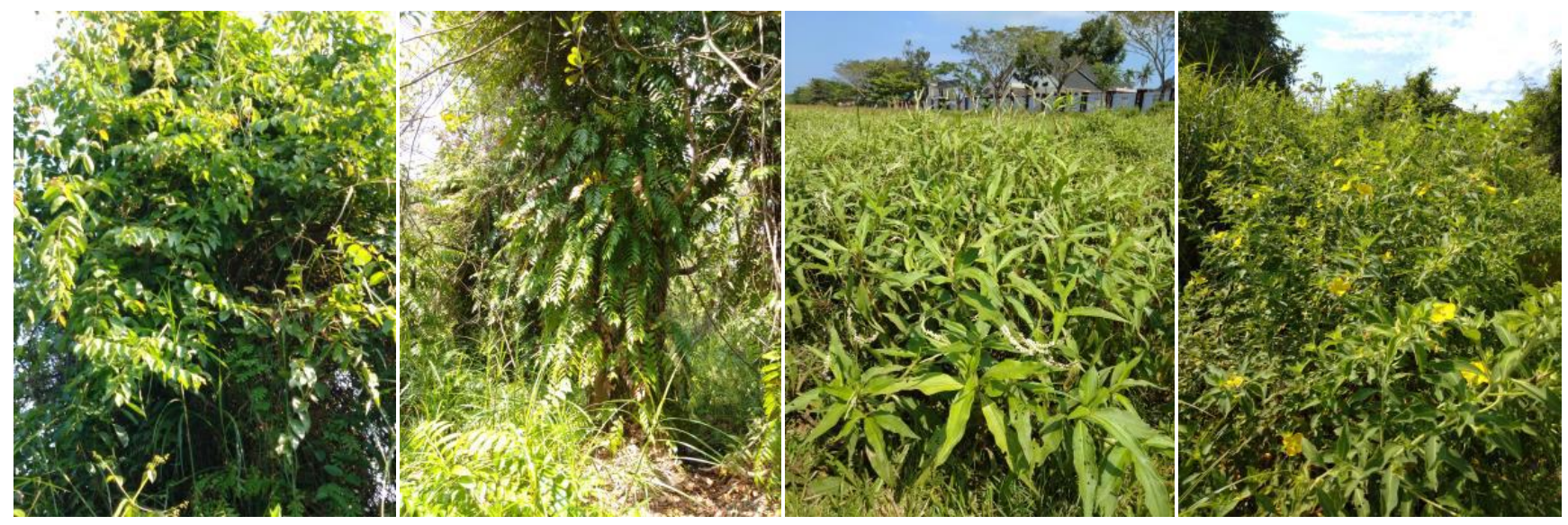

Figure 4. From left to right: Uncaria acida covering Alstonia spatulata; Stenochlaena palustris covering Campnosperma coriaceum; Persicaria attenuata dominating an open area; Ludwigia peruviana dominating an open area.
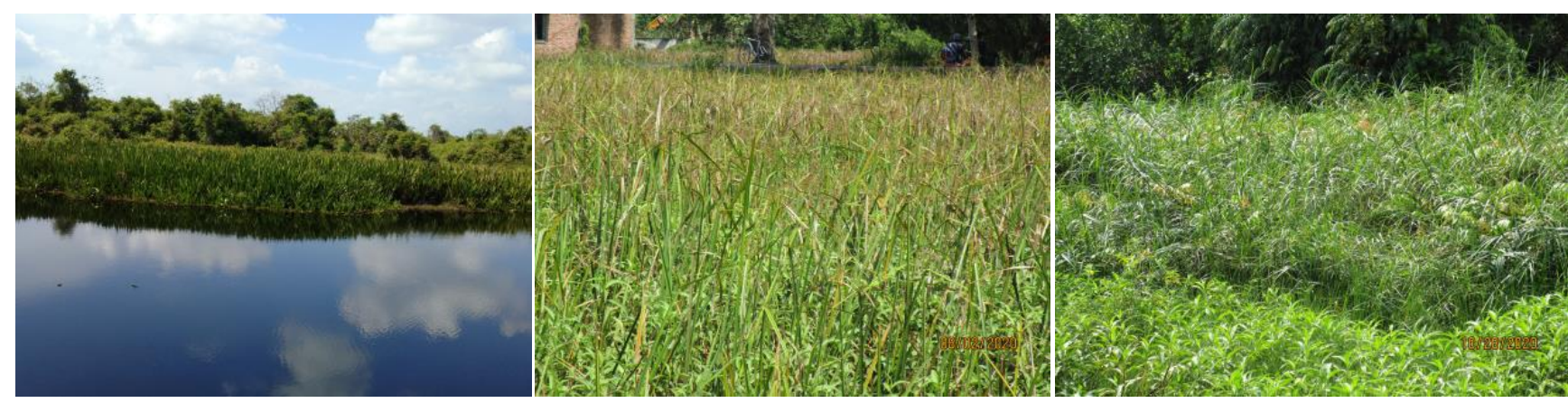

Figure 5. From left to right. Crinum asiaticum abundant in some locations; Actinoscirpus grossus dominating an open area; Scleria ciliaris, in the middle of the picture in the right, and Persicaria attenuata, in the front, dominating an open area 
Another sedge, Actinoscirpus grossus or Scirpus grossus, was also found abundant in some open space in the swamp with an importance value of 10.58 , ranked $5^{\text {th }}$ among the ground cover species (Table 2; Figure 5). This perenial sedge is native to tropical Asia, tropical Australia (Flora Malesiana 2020), and usually grows in swampy areas, marshes, ditches, and it may be dominant locally (Soerjani et al. 1987). It is considered a pan-tropical weed in the rice fields, drainage and irrigation canals, riverbanks (Majrashi et al. 2013). Beside having negative impacts, this species also has positive impacts for the environmenment because of its ability to absorb $\mathrm{Al}$ and Fe from the water, so it has been used for phytoremediation of contaminated water (Al-Badawi et al. 2015; Ismail et al 2019).

In some open wet places in this study site, Persicaria attenuata was found abundant, forming almost a pure stand (Figure 4). It had an importance value of 11.31, ranked $4^{\text {th }}$ among the ground cover species (Table 2). This species with common English names of smartweed, watersmart weed, hairy knotweed is widespread in the tropics and subtropics of Africa, Asia and Australia, growing along the edge of swamp, dams, lagoons, bilabong and rivers, which may form thick, pure stand (Calvert and Liessmann 2014). Although this species was dominant in some open spaces in the swamp, this species was not listed as weeds of rice, while another spcies of he same genus, Polygonum barbatum (a synonym of Persicaria barbata) was listed, but this species was of minor concern (Soerjani et al. 1987). With its ability to form thick pure stand, Persicaria attenuata can withstand the invasion of alien species (Calvert and Liessmann 2014).

Another forb found abundant in the study site was Crinum asiaticum (Figure 5), ranked $6^{\text {th }}$ among the ground cover species (Table 2). It is native to Indian Ocean, tropical and subtropical Asia to Southwest Pacific and can grow in many types of habitat, i.e., sandy shores, freshwater swamps, mangroves, savannah to desert areas, up to $700 \mathrm{~m}$ above sea level (Sukarya 2013). This species was also found in lowland wetland in Morang District of Nepal (Koirala and Jha 2011). Like A.grossus, C. asiaticum can accumulate metals as reported by Amin et al (2015) that in Kawasi wetland on Obi Island, North Maluku Province, $C$. asiaticum was the most dominant species and accumulated the highest concentration of metals both in roots and shoots.

\section{Palm}

A species of palm found abundant in some places in the swamp was Nypa fruticans. The genus Nypa was one of the oldest palms whose fossil records show that this genus was found in many parts of the world, but its current distribution is limited to tropical areas (Gomez-Navarro 2009). Nypa fruticans is naturally distributed from Asia extending to Europe, Africa, and America, but it is now found only in the tropical Indo-West Pacific region, from Sri Lanka through Asia to Northern Australia and the Western Pacific islands (Hossain and Islam 2015). Although this species has many benefits for society (Hossain and Islam 2015), it has become an invasive weed species in West Africa since its introduction in the early twentieth century (Noblik et al. 2018). Its superior root system enables this species to exploit nutrient in the environment and its buoyant seeds lead to its widespread distribution in Nigeria, threatening mangrove ecosystem (Numbere 2019).

\section{Conclusion and recommendation for future studies}

This study has shown the dominant plant species in the swamp forest, consisting of trees, shrubs and herbs. However, due to its small number of plots, this study has not completely described the flora of the swamp forest. Further studies with more sample plots located properly by considering the spatial heterogeneity of habitat will give more comprehensive description of the structure and composition of the swamp forest.

Studies on specific topic can be conducted to add the ecological understandings and help biodiversity conservation. For example, the abundance of climbers which cover the tree crowns should be studied to give us better understanding on the effects of climbers on trees. Climbers are competitors of trees in getting sunlight from above the crowns and in getting nutrients from the soil, and therefore, they may slow the succession rate of the seconday forest (Paul and Yavitt 2011). Another topic is the invasivsion of alien species, Ludwigia peruviana. According to the Indonesian law, the objective of strict nature reserve (cagar alam) is to preserve the specific natural ecosystem. Currently the ecosystem in the study site as a strict nature reserve has been drastically changed by illegal cutting and alien species invasion, and therefore should be restored.

\section{ACKNOWLEDGEMENTS}

We express our gratitude to the Provincial Government of Bengkulu, Indonesia which has provided fund for this study. During the field survey, we got assistance from Rahayu, Muharif, Taufiq, Gad, Amegi, Willy, Restu, Gad, Dio, Crisnal, Jelly and Zen. We are grateful to them.

\section{REFERENCES}

Adam JH, Wilcox CC, Swaine MD. 1992. The ecology and distribution of Bornean Nepenthes. J Trop For Sci 5 (1): 13-25.

Al-Baldawi IA, Abdullah SRS, Anuar N, et al. 2015.Phytodegradation of total petroleum hydrocarbon (TPH) in diesel-contaminated water using Scirpus grossus. Ecol Eng 74: 463-473.

Amin R, Edraki M, Mulligan R, Gultom TH. 2015. Chromium and nickel accumulation in the macrophytes of the Kawasi wetland on Obi Island, North Maluku Province, Indonesia. Aust J Bot 63 (7): 549553.

Australian tropical rain forest plants. http: //www.anbg.gov.au/cpbr/cdkeys/RFK7/key/RFK7/Media/Html/index_rfk.htm

Baas P, Fujii T. 2020. Earlier accounts of driftwood of Alstonia spatulata (Apocynaceae). Gard Bull Sing 72 (1): 131-132.

Backer CA, van Der Brink RCB. 1963. Flora of Java. Volume I. NVP Noordhoff, Groningen.

Backer CA, van Der Brink RCB. 1965. Flora of Java. Volume II. NVP Noordhoff, Groningen.

Backer CA, van Der Brink RCB. 1968. Flora of Java. Volume III. NVP Noordhoff, Groningen. 
Barua IC, Deka J, Devi M, Deka RL, Moran J. 2017. Weeds as emerging threat to biodiversity: a consequence of spread of Ludwigia peruviana in Dhansiri and Kopili catchment areas of Assam, North East India. Curr Sci 112 (9): 1904-1914.

Cahyono DB, Roini C, Tamalene MN. 2019. Karakteristik habitat tumbuhan kantong semar (Nepenthes sp.) di pulau Halmahera. Techno 8 (1): 233-141. [Indonesian]

Calvert G, Liessmann L. 2014. Wetland Plants of the TownsvilleBurdekin Flood Plain. Lower Burdekin Landcare Association Inc., Ayr.

Chai PPK. 2016. Midin (Stenochlaena palustris), the popular wild vegetable of Sarawak. Utar Agric Sci J 2 (2): 18-20.

Chandrasena N, Pinto L, Sim R. 2002. Reclaiming Botany Wetlands, Sydney through integrated management of Ludwigia peruviana and other weeds. In: Spafford JH, Dodd J, et al. (eds.). 13th Australian Weeds Conference Papers \& Proceedings, Perth, Australia.

Chong KY, Lim RC, Loh JW, et al. 2018. Rediscoveries, new records, and the floristic value of the Nee Soon freshwater swamp forest, Singapore. Gard Bull Sing 70 (1): 49-69.

Clews E, Corlett RT, Ho JKI, et al. 2018. The biological, ecological and conservation significance of freshwater swamp forest in Singapore. Gard Bull Sing 70 (1): 9-31.

Davison GWH, Cai Y, Li TJ, Lim WH. 2018. Integrated research, conservation and management of Nee Soon freshwater swamp forest, Singapore: hydrology and biodiversity. Gard Bull Sing 70 (1): 1-7

Eijk P van, Pieter Leenman P, Wibisono ITC, Giesen W. 2009. Regeneration and restoration of degraded peat swamp forest in Berbak NP, Jambi, Sumatra, Indonesia. Malayan Nat J 61 (3): 223 241

Eviona Y. 2016. Dampak Kerusakan Kawasan Cagar Alam Danau Dusun Besar Terhadap Aspek Sosial Ekonomi Petani di Sekitar Danau Dendam Tak Sudah Kota Bengkulu. [Thesis]. Universitas Andalas, Padang. [Indonesian]

Flora Malesiana 2020. http: //portal.cybertaxonomy.org/flora-malesiana

Giesen W, Wijedasa LS, Page SE. 2018. Unique Southeast Asian peat swamp forest habitats have relatively few distinctive plant species. Mires Peat 22 (1): 1-13, http: //www.mires-and-peat.net

Gomez-Navarro C, Jaramillo C, Herrera F, Wing SL, Callejas R. 2009. Palms (Arecaceae) From A Paleocene Rainforest of Northern Colombia. Aust J Bot 96: 1300-1312.

Hidayat S, Helmanto H, Dodo, Purnomo DW, Supriyatna I. 2018. Habitat of Nepenthes spp. in the area of Sampit Botanic Gardens, Central Kalimantan, Indonesia. Biodiversitas 19 (4): 1258-1265

Ho JKI, Ramchunder SJ, Memory A, et al. 2019. Native and introduced fish community structure in a freshwater swamp forest: Implications for conservation and management. Aquatic Conserv: Mar Freshw Ecosyst 29 (1): 47-58

Hossain MF, Islam MA. 2015. Utilization of Mangrove Forest Plant: Nipa Palm (Nypa fruticans Wurmb.). Amer J Agric For 3 (4): 156-160.

International Plant Names Index (https: //www.ipni.org/n/22504-2)

Invasive species compendium (https: //www.cabi.org/isc)

Ismail NI, Abdullah SRS, Idris M. et al. 2019. Simultaneous bioaccumulation and translocation of iron and aluminium from mining wastewater by Scirpus grossus. Desalin Water Treatm 163: $133-142$.

Koirala U, Jha S. 2011. Macrophytes of the lowland wetlands in Morang district, Nepal. Nepalese J Biosci 1: 131-139.

Majrashi A, Bakar BB, Khandaker MM. et al. 2013. Fractal analysis of rhizome growth patterns of Scirpus grossus L. on peat and paddy soils. Bulg J Agric Sci 19: 1319-1326.

Mardhiana, Parto Y, Hayati R, Priadi DP. 2012. The characteristics and abundance of Nepenthes of nutrient-poor habitats. Jurnal Lahan Suboptimal 1 (1): 50-56. [Indonesian]

Margurran, A. 2004. Measuring Biodiversity. Blackwell Science Publishing, Oxford, UK.

Moody K, Munroe CE, Lubigan RT, Paller EC Jr, 1984. Major weeds of the Philippines., Los Baños, Philippines: Weed Science Society of the Philippines, University of the Philippines at Los Baños.

Mueller-Dombois D, Ellenberg H. 1974. Aims and Methods in Vegetation Ecology. John Willey \& Sons. New York.

Noblick L, Lima JMT, Valdes IR. 2018. Nypa fruticans in the Western Atlantic: Potential for Recolonization? Palms 62 (4): 175-184.
Noda K, Teerawatsakul M, Prakongvongs C. Chaiwiratnukul L. (1994). Major Weeds in Thailand. National Weed Science Research Institute Project, (Revised 2nd edition).

Novianti V, Choesin DN, Iskandar DT, Suprayogo, D. 2017. Plant species from coalmine overburden dumping site in Satui, South Kalimantan, Indonesia. J. Degrade. Min. Land Manage. 4 (4): 927-936.

Numbere AO. 2019. Impact of Invasive Nypa Palm (Nypa Fruticans) on Mangroves in Coastal Areas of the Niger Delta Region, Nigeria. In: Makowski C, Finkl CW (eds.), Impacts of Invasive Species on Coastal Environments. Coastal Research Library 29.

Paul GS, Yavitt JB. 2011. Tropical Vine Growth and the Effects on Forest Succession: A Review of the Ecology and Management of Tropical Climbing Plant. Bot Rev 77: 11-30.

Permono AR. 2007. Analisis struktur dan komposisi vegetasi tingkat pohon dan tiang hutan rawa air tawar di kawasan Cagar Alam Danau Dusun Besar (CADDB) kota bengkulu. [Hon. Thesis], Fakultas Pertanian, Universitas Bengkulu, Bengkulu. [Indonesian]

Plants of the World online. http: //plantsoftheworldonline.org/?

Rajapakse R, Chandrasena N, Marambe B, Amarasinghe L. 2011. Planning for effective weed management: lessons from Sri Lanka. 23rd Asian-Pacific Weed Science Society Conference the Sebel Cairns, 26-29 September 2011

Rastogi J, Rawat DS, Chandra S. 2015. Diversity of invasive alien species in Pantnagar flora. Tropical Plant Res 2 (3): 282-287.

Rizqiani S, Ariyanti NS, Sulistijorini. 2018. Diversity of Lowland Nepenthes (Pitcher Plants) in Bangka Belitung Islands. IOP Conf Ser Earth Environ Sci 197: 012021. DOI: 10.1088/1755$1315 / 197 / 1 / 012021$

Slik JWF. 2009 onwards. Plants of Southeast Asia. http: //www.asianplant.net/

Soerjani M, Kostermans AJGH, Tjitrosoepomo G (eds.). 1987. Weeds of Rice in Indonesia. Balai Pustaka, Jakarta.

Subarnas, A. 2001. Uncaria acida (Hunt.) Roxb.. In: van Valkenburg JLCH, Bunyapraphatsara N (eds.): Plant Resources of South-East Asia No 12 (2): Medicinal and Poisonous Plants 2. PROSEA Foundation, Bogor, Indonesia.

Sudirja R, Hindersah R. 2009. Kesesuaian lahan tanaman keras di Kawasan Konservasi Danau Dendam Tak Sudah Kota Bengkulu. Prosiding Talk Show dan Seminar Nasional Peran Informasi Geospasial Pertanahan Bandung, 4 Maret 2009. [Indonesian]

Sukarya DG (eds.). 2013. 3500 Plant Species of the Botanic Gardens of Indonesia. PT Sukarya \& Sukarya Pandetama, Indonesia

Tan SY, Koh CY, Siow HJM, 2013. 100 Common Vascular Plants of the Nee Soon Swamp Forest, Singapore. Raffles Museum of Biodiversity Research and National University of Singapore, Singapore.

Tasori, Wiryono, Sulistyo B. 2014. Kajian kerusakan pada cagar Alam Danau Dusun Besar, Bengkulu. Naturalis 3 (1): 75-83.

Teo SP. 2001. Alstonia spatulata Bl. In: van Valkenburg JLCH, Bunyapraphatsara N (eds.): Plant Resources of South-East Asia No 12 (2): Medicinal and Poisonous Plants 2. PROSEA Foundation, Bogor, Indonesia.

The Plant List. http: //www.theplantlist.org/

Theilade I, Schmidt L, Chhang P, Mcdonald J. 2011. Evergreen swamp forest in Cambodia: floristic composition, ecological characteristics, and conservation status. Nordic J Bot 29: 71-80.

Toth LA. 2010. Restoration response of relict broadleaf marshes to increased water depths. Wetlands30: 263-274.

Turner IM. 2018. Notes on the genus Uncaria (Rubiaceae) in Singapore. Gard Bull Sing 70 (1): 9-12.

Usmadi D, Witono JR, Lestari R, et al. 2020. Plant diversity and composition at three Imperata grasslands in Bogor, Katingan, and Kupang, Indonesia. Biodiversitas 21 (6): 2804-2813.

Wahyuni I, Sulistijorini, Tjitrosoedirdjo S. 2015. Inventory of Invasive Plant Species at Bukit Duabelas National Park and the Vicinity, Jambi, Sumatra Proceedings Papers of International Conference on Biosciences (ICoBio): 52-61. 5icobio.event.ipb.ac.id

Whitmore TC. 1984. Tropical Rain Forests of the Far East. Clarendon Press, Oxford.

Whitten AJ, Damanik SJ, Anwar J, Hisyam N. 1984. The Ecology of Sumatra. Gadjah Mada University Press, Yogyakarta.

Yusuf R, Purwaningsih. 2009. Vegetation study on freshwater swamp forest of Rimbo Panti Nature Reserve, West Sumatra. Berita Biologi 9 (5): 491-508. [Indonesian] 